\title{
Mixed reviews on removing fallopian tubes to prevent ovarian cancer
}

$\mathrm{F}$ ollowing an extensive educational campaign, surgeries to remove fallopian tubes as a way to prevent ovarian cancer have increased by $16 \%$ in Canada over the last two years, despite resistance from some experts who say there's not enough evidence for doctors to recommend the surgery.

"The problem is that the evidence will take 20 years to emerge," says Dr. John Thiel, clinical professor of obstetrics at the University of Saskatchewan in Regina and a vocal opponent of the surgery, known as a salpingectomy. "Sure, it's a cool, interesting idea that perhaps you could remove the fallopian tubes and reduce risk of this terrible disease, and this would be a great intervention if it works. But if you don't have evidence that it works, how can you recommend it?"

Across the country, doctors performed 34611 salpingectomies in 20112012, up from 28999 in 2008-2009. In British Columbia, 3806 were performed during 2008-2009. By 2011-2012, that number had increased to 6133 .

In 2011, the Society of Gynecologic Oncology of Canada endorsed a prevention campaign from BC's Ovarian Cancer Research Program (OVCARE). Beginning in September 2010, the program asked the province's gynecologists to talk to patients about the removal of their fallopian tubes during hysterectomies and in place of tubal ligation for women seeking irreversible contraception.

OVCARE developed the strategy based on research from multiple centres over the previous decade that indicated $70 \%$ of high-grade serous ovarian cancers actually arise from the fallopian tubes.

According to the $\mathrm{BC}$ program, the surgery will reduce the risk of ovarian cancer for women in the general population already considered at relatively low risk. For women who carry the

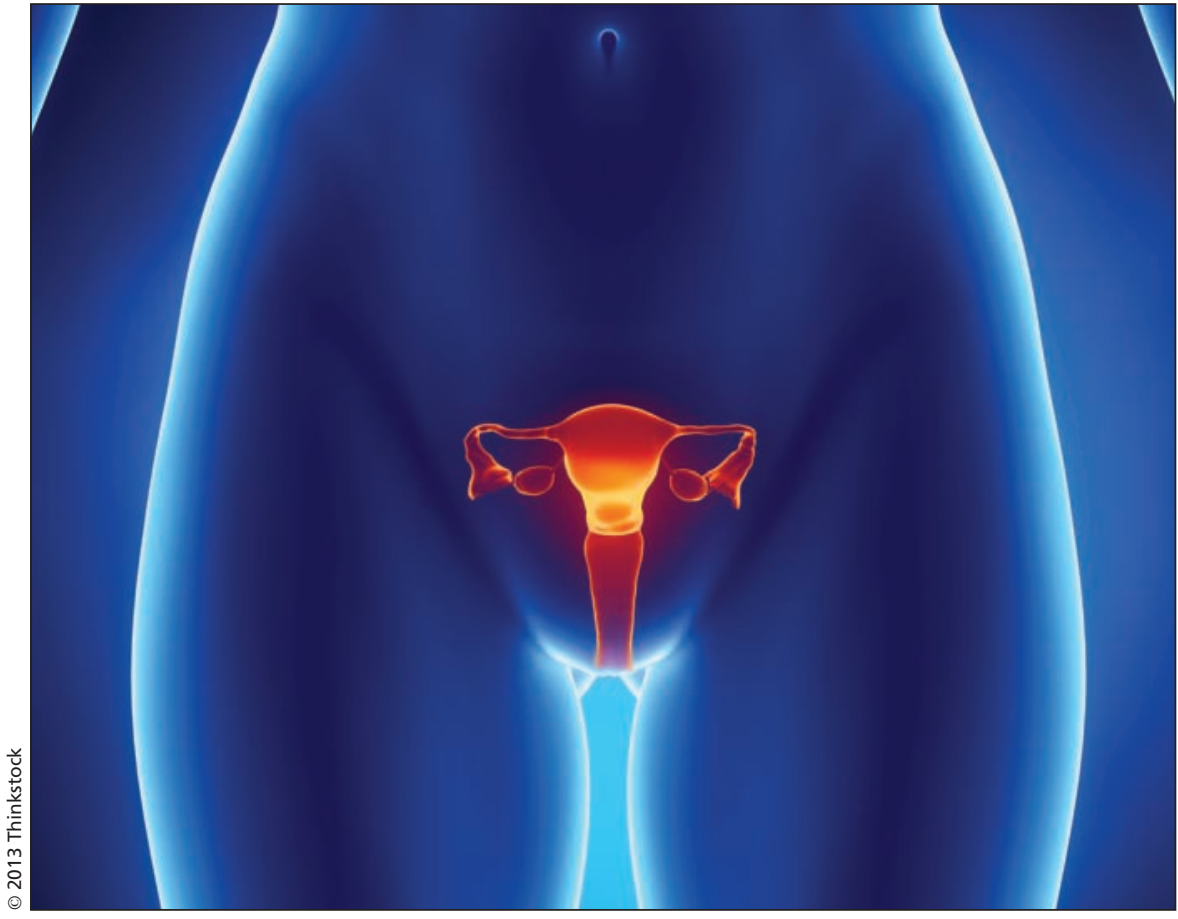

Research from multiple centres over the previous decade indicates that $70 \%$ of highgrade serous ovarian cancers arise from the fallopian tubes.

BRCA1 or BRCA2 gene mutations and are thus at increased lifetime risk of breast and ovarian cancer, OVCARE continues to recommend risk-reducing surgery to remove both the ovaries and fallopian tubes.

"We believe the evidence supporting the fallopian tube as the site of origin of the most common type of ovarian cancer is indisputable [and that] these simple changes in clinical and surgical practice will have an important impact on the number of ... cases over the next two decades," OVCARE's team of doctors wrote (Clin Adv Hem Oncol 2012; 10:303). "We have an opportunity to ... embrace a new surgical paradigm for ovarian cancer prevention."

The Society of Gynecologic Oncology of Canada has listed an ovarian cancer prevention study focused on removal of the fallopian tubes as a research priority.

The prevention recommendations are backed by solid research, says Dr. Mark Heywood, past-president of the Society of Obstetricians and Gynaecologists of Canada. "Multiple publications discuss high-grade serous carcinoma being of fallopian tube origin in many cases, and it's based on that evidence that we're making the recommendation...."

But like Thiel, Dr. Robert Lotocki, a gynecologic oncologist at St. Boniface Hospital in Winnipeg, Manitoba, believes doctors don't know enough about risk and benefits to make informed recommendations.

"Women who have a standard tubal ligation still get benefits in terms of protection from serous epithelial cancer," he says. "But whether they get more protection from removing the tubes hasn't been defined, and neither has the risk."

Researchers and clinicians such as Dr. Jessica McAlpine, OVCARE's gynaecologic tissue bank director, aren't 
suggesting women have surgery solely to remove their fallopian tubes.

"We're recommending salpingectomy be discussed at the time of pelvic or abdominal surgery," says McAlpine, also an associate professor in the Department of Obstetrics and Gynaecology at the University of British Columbia in Vancouver. "Why would you not offer this risk reduction procedure when someone is already having abdominal surgery?"

OVCARE researchers have embarked on a long-term study to discover whether removing fallopian tubes will definitively reduce the incidence of ovarian cancer in $\mathrm{BC}$ over the next 20 years.

Heywood acknowledges that a randomized controlled trial is the gold standard but says "we believe we can get the same evidence through a population health basis, by following people who've had their tubes removed, and comparing them with those that have not and the incidence of high grade serous cancer over time. If we're right, everyone who's had the procedure will have had the benefit of reduced risk, but if we're wrong, there's probably little to no downside."
Removing fallopian tubes is not a difficult procedure and is well within the skill sets of gynecologic surgeons, adds McAlpine. "Concerns about potential hormonal impact or increased complications associated with the procedure are being addressed by the OVCARE team and others. Pending publications and presentations regarding both of these issues suggest no negative consequences." — Lauren Kramer, Vancouver, BC

CMAJ 2013. DOI:10.1503/cmaj.109-4475 